\title{
GLOBAL DIMENSION OF TRIANGULAR ORDERS OVER A DISCRETE VALUATION RING
}

\author{
VASANTI A. JATEGAONKAR
}

\begin{abstract}
We characterize triangular $R$-orders of finite global dimension in $n \times n$ matrix rings over the quotient field of DVR $R$ and obtain a precise upper bound for their global dimension, viz. $n-1$. We also characterize triangular $R$-orders of highest global dimension.
\end{abstract}

Introduction. Throughout $R$ is a discrete valuation ring (DVR) with the unique maximal ideal $m$, generated by $t$, and quotient field $K$. An $R$ order $\Lambda$ in $M_{n}(K)$ is an $R$-subalgebra of $M_{n}(K)$ which is finitely generated as an $R$-module and spans $M_{n}(K)$ over $K . \Lambda$ is tiled if $\Lambda$ contains $n$ orthogonal idempotents. After a conjugation, if necessary, we may assume that $e_{i i} \in \Lambda$, where $e_{i j}$ are the usual matrix units in $M_{n}(K)$. Then $\Lambda$ is of the form $\Lambda=\left(m^{\lambda_{i i}}\right)$, where $\lambda_{i j} \in Z$. If $\lambda_{i j}=0$ whenever $i \leqq j$ then $\Lambda$ is called a triangular $R$-order. We set $\Omega_{n}=\left(m^{\mu_{i j}}\right) \subseteq M_{n}(K)$, where $\mu_{i j}=0$ whenever $i \leqq j$ and $\mu_{i j}=i-j$ otherwise.

The main result of this paper is the following

THEOREM. Given a triangular $R$-order in $M_{n}(K)$, the following are equivalent: (1) gl. $\operatorname{dim} . \Lambda<\infty$, (2) $\Omega_{n} \subseteq \Lambda$, (3) gl. dim. $\Lambda \leqq n-1$.

This result was conjectured by R. B. Tarsey [5]. In the same paper, Tarsey constructs a triangular $R$-order in $M_{n}(K)$ of global dimension $n-1$. Hence the bound in our theorem is best possible. We also give a characterization of triangular $R$-orders of highest global dimension. Using this we construct examples of successive triangular $R$-orders in $M_{2 n+1}(K)$ whose global dimensions differ exactly by $n$. This disproves a conjecture in R. B. Tarsey [5].

The main results of this paper were announced in [1].

After this paper was completed the author received a preprint of [6] from R. B. Tarsey in which he has independently obtained $(1) \Leftrightarrow(2)$ in the above theorem; however, his methods yield a bound $2 n-4$ rather than $n-1$.

Received by the editors May 28, 1971.

AMS (MOS) subject classifications (1970). Primary 18G20; Secondary 16A62.

Key words and phrases. Global dimension, order, tiled order, triangular tiled order, discrete valuation ring, Dedekind domains.

(c) American Mathematical Society 1973 
The proof of the theorem depends on the following lemmas.

LEMMA 1. If $\Lambda$ is an $R$-order in an algebra over the quotient field of DVR $R$ and if $J(\Lambda)$ is the Jacobson radical of $\Lambda$, then $\mathrm{gl}$. dim. $\Lambda=1+$ $\mathrm{hd}_{\Lambda} J(\Lambda)$.

Proof. Silver [4, Corollary 4.6].

LEMmA 2. If $\Lambda$ is any ring, then $\mathrm{rt}$. gl. $\operatorname{dim} . \Lambda=1+\sup _{I}\{$ hd $I\}$ where supremum is taken over right ideals of $\Lambda$, unless $\Lambda$ is semisimple.

Proof. Well known [3].

Henceforth, we shall fix a positive integer $n$ and unless stated otherwise, $\Lambda=\left(m^{\lambda_{i j}}\right)$ will denote a triangular $R$-order in $M_{n}(K) ; P_{i}$ and $J_{i}$ denote the $i$ th row of $\Lambda$ and its Jacobson radical respectively. We shall always treat $P_{i}$ and $J_{i}$ as canonical submodules of the first row of $\Lambda$. This makes expressions like $P_{i}+P_{j}$ unambiguous. Observe that if $\lambda_{i, i-1} \supsetneqq 0$ for $2 \leqq i \leqq n$, then $J(\Lambda)$ is obtained from $\Lambda$ by replacing the diagonal entries $R$ by $m$.

Let $e=\sum_{i=1}^{n-1} e_{i i}$, where $e_{i i}$ are the usual idempotents in $\Lambda$. We shall interchangeably treat $e \Lambda e$ as top $(n-1) \times(n-1)$ corner of $\Lambda$ or as a triangular order in $M_{n-1}(K)$. Let $\mathscr{F}: \bmod -\Lambda \rightarrow \bmod -e \Lambda e$ and $\mathscr{G}: \bmod -e \Lambda e \rightarrow$ $\bmod -\Lambda$ be the functors defined by $\mathscr{F}(M)=M e, M \in \bmod -\Lambda$ and $\mathscr{G}(N)=$ $N \otimes_{e \Lambda e} e \Lambda$ where $e \Lambda=\oplus_{i=1}^{n-1} P_{i}$. We shall have repeated occasions to use these functors.

Lemma 3. (a) $\mathscr{F}$ and $\mathscr{G}$ are exact additive functors. $\mathscr{F} P_{1}, \cdots, \mathscr{F} P_{n-1}$ are principal projectives of $e \Lambda e . e \Lambda$ is a progenerator in $e \Lambda e-\bmod$ and $J(e \Lambda e)$ is canonically isomorphic with $\oplus_{i=1}^{n-1} \mathscr{F} J_{i}$.

(b) $\mathscr{G} \mathscr{F} P_{i} \cong P_{i}$ for $1 \leqq i \leqq n-1$.

(c) For every right $e \Lambda e-$ module $N$ and right $\Lambda$-module $M$, we have,

$$
\operatorname{hd}_{\Lambda} \mathscr{G} N \leqq \operatorname{hd}_{e \Lambda e} N, \quad \operatorname{hd}_{e \Lambda e} \mathscr{F} \mathscr{G} \mathscr{F} M \leqq \mathrm{hd}_{\Lambda} \mathscr{G} \mathscr{F} M .
$$

Further, if $\mathscr{G} \mathscr{F} M \cong M$ then $\mathrm{hd}_{\Lambda} M=\mathrm{hd}_{e \Lambda e} \mathscr{F} M$.

Proof. The first part is clear. For part (b) we observe that $e_{e \Lambda e} e \Lambda$ is projective (hence flat) and $\mathscr{F} P_{i}$ is isomorphic to a right ideal of $e \Lambda e$, therefore

$$
0 \rightarrow \mathscr{F} P_{i} \otimes_{e \Lambda e} e \Lambda \rightarrow e \Lambda e \otimes_{e \Lambda e} e \Lambda
$$

is exact. Hence $\mathscr{G} \mathscr{F} P_{i} \cong\left(\mathscr{F} P_{i}\right) e \Lambda$. The last two entries in $P_{i}$ are equal for $1 \leqq i \leqq n-1$. So, $\left(\mathscr{F} P_{i}\right) e \Lambda=P_{i}$.

The first inequality in (c) is clear since $e \Lambda_{\Lambda}$ is projective. Now, suppose $\operatorname{hd}_{\Lambda} \mathscr{G} \mathscr{F} M=l<\infty$. Let

$$
\cdots \longrightarrow M_{i} \stackrel{d_{i}}{\longrightarrow} M_{i-1} \longrightarrow \cdots-M_{1} \stackrel{d_{1}}{\longrightarrow} M_{0} \stackrel{d_{0}}{\longrightarrow} \mathscr{F} M \longrightarrow 0
$$


be a projective resolution of $\mathscr{F} M$ over $e \Lambda e$. Since $e \Lambda e$ is semiperfect and $\mathscr{F} P_{1}, \cdots, \mathscr{F} P_{n-1}$ are the only principal projectives of $e \Lambda e$, therefore each $M_{i} \cong \oplus_{j=1}^{n-1} \mathscr{F} P_{j}^{k_{i j}}$, where the $k_{i j}$ are (possibly empty) sets. Clearly,

$$
\begin{aligned}
\cdots \longrightarrow \mathscr{G}_{M_{i}} \stackrel{d_{i} \otimes 1}{\longrightarrow} \mathscr{G}_{M_{i-1}} \stackrel{d_{i-1} \otimes 1}{\longrightarrow} \cdots \longrightarrow \\
\mathscr{G}_{M_{1}} \stackrel{d_{1} \otimes 1}{\longrightarrow} \mathscr{G}_{M_{0}} \stackrel{d_{0} \otimes 1}{\longrightarrow} \mathscr{G} \mathscr{F} M \longrightarrow 0
\end{aligned}
$$

is a projective resolution of $\mathscr{G} \mathscr{F} M$ over $\Lambda$. Since $\mathrm{hd}_{\Lambda} \mathscr{G} \mathscr{F} M=l<\infty$,

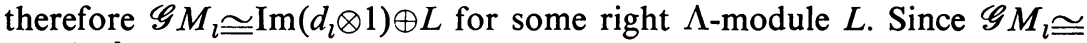
$\oplus_{j=1}^{n-1} P_{j}^{k_{l j}}$ and $\Lambda$ is semiperfect, therefore by the decomposition theorem [2, Theorem 3] and Krull-Schmidt-Azumaya theorem, $\operatorname{Im}\left(d_{l} \otimes 1\right) \cong$ $\oplus_{j=1}^{n-1} P_{j}^{k^{\prime}{ }^{\prime} j}$ for some (possibly empty) sets $k_{l j}^{\prime}$. This shows that $\mathscr{F} \operatorname{Im}\left(d_{l} \otimes 1\right)$ is a right $e \Lambda e$-projective module. Now,

$$
0 \rightarrow \mathscr{F} \operatorname{Im}\left(d_{l} \otimes 1\right) \rightarrow \mathscr{F} \mathscr{G}_{M_{l-1}} \rightarrow \cdots \rightarrow \mathscr{F} \mathscr{G}_{M_{0}} \rightarrow \mathscr{F} \mathscr{G} \mathscr{F} M \rightarrow 0
$$

is a projective resolution of $\mathscr{F} \mathscr{G} \mathscr{F} M$ over $e \Lambda e$, which yields

$$
\operatorname{hd}_{e \Lambda e} \mathscr{F} \mathscr{G} \mathscr{F} M \leqq l .
$$

The last assertion follows from above two inequalities.

LEMmA 4. If $\lambda_{i, i-1} \supsetneqq 0$ for $2 \leqq i \leqq n$ and $\lambda_{n, n-1}=1$, then $\mathscr{G F} J_{i} \cong J_{i}$ for $i \neq n-1$.

Proof. The proof is similar to that of part (b) of Lemma 3.

LemmA 5. If gl. dim. $\Lambda<\infty$, then $\lambda_{2,1} \leqq 1$ and $\lambda_{n, n-1} \leqq 1$.

Proof. Suppose $\lambda_{2,1} \geqq 2$. We have exact sequences,

$$
\begin{aligned}
& 0 \longrightarrow t P_{1} \cap P_{2} \stackrel{\phi_{1}}{\longrightarrow} t P_{1} \oplus P_{2} \stackrel{\theta_{1}}{\longrightarrow} J_{1} \longrightarrow 0, \\
& 0 \longrightarrow t^{\lambda_{2,1}-1} P_{1} \cap P_{2} \stackrel{\phi_{2}}{\longrightarrow} t^{\lambda_{2,1}-1} P_{1} \oplus P_{2} \stackrel{\theta_{2}}{\longrightarrow} t^{\lambda_{2,1}-1} P_{1}+P_{2} \longrightarrow 0,
\end{aligned}
$$

where $\phi_{i}(x)=(x, x)$ and $\theta_{i}(x, y)=x-y$ for $i=1,2$. Since $J_{1}$ is not projective, $t P_{1} \cap P_{2} \cong t^{\lambda_{2,1}-1} P_{1}+P_{2}$ and $t^{\lambda_{2,1}-1} P_{1} \cap P_{2} \cong J_{1}$, therefore hd $J_{1}=\infty$, contrary to our hypothesis. Hence $\lambda_{2,1} \leqq 1$. Similarly, looking at appropriate left $\Lambda$-modules we get $\lambda_{n, n-1} \leqq 1$.

LEMMA 6. (a) If $\Omega_{n} \subseteq \Lambda$, then $\Omega_{n-1} \subseteq e \Lambda e$.

(b) If $\lambda_{l, l-1}=0$ for some $l$ and if $f=\sum_{i=1 ; i \neq l}^{n} e_{i i}$, then $\Omega_{n} \subseteq \Lambda$ iff $\Omega_{n-1} \subseteq$ $f \Lambda f \subseteq M_{n-1}(K)$.

PROOF. The first part is clear since $\Omega_{n-1}=e \Omega_{n} e$. Now assume $\Omega_{n} \subseteq \Lambda$. 
Since

$$
\lambda_{i, j} \leqq \lambda_{i, l}+\lambda_{l, l-1}+\lambda_{l-1, j}=\lambda_{i, l}+\lambda_{l-1, j},
$$

therefore, if $i_{\neq} l \supsetneqq j$ then $\lambda_{i, j} \leqq(i-l)+(l-1-j)=i-1-j$. It follows that $\Omega_{n-1} \subseteq f \Lambda f$. The remaining case is similarly dealt with.

Proposition 1. If $\lambda_{i, i-1 \neq} \neq 0$ for $2 \leqq i \leqq n$ and if $\lambda_{n, n-1}=1$, then $\operatorname{gl}$. dim. $\Lambda<$ $\infty$ if and only if gl. dim. $e \Lambda e<\infty$. Further, if gl. $\operatorname{dim} . \Lambda=\alpha<\infty$ and if gl. $\operatorname{dim} e \Lambda e=\beta<\infty$ then $\beta \leqq \alpha \leqq \beta+1$.

Proof. By Lemma $4, J_{i} \cong \mathscr{G} \mathscr{F} J_{i}$ for $i \neq n-1$. Clearly, $\mathscr{F} \mathscr{G} \mathscr{F} J_{n-1} \cong$ $\mathscr{F} J_{n-1}$. Therefore, by Lemma 3 ,

$$
\operatorname{hd}_{\Lambda} J_{i}=\operatorname{hd}_{e \Lambda e} \mathscr{F} J_{i} \text { for } i \neq n-1
$$

and $\operatorname{hd}_{\Lambda}\left(\mathscr{F} J_{n-1}\right) e \Lambda=\mathrm{hd}_{\Lambda} \mathscr{G} \mathscr{F} J_{n-1}=\mathrm{hd}_{e \Lambda e} \mathscr{F} J_{n-1}$. Clearly, $\left(\mathscr{F} J_{n-1}\right) e \Lambda+$ $P_{n}=J_{n-1},\left(\mathscr{F} J_{n-1}\right) e \Lambda \cap P_{n}=J_{n}$. Hence,

$$
0 \longrightarrow J_{n} \stackrel{\phi}{\longrightarrow}\left(\mathscr{F} J_{n-1}\right) e \Lambda \oplus P_{n} \stackrel{\theta}{\longrightarrow} J_{n-1} \longrightarrow 0
$$

is exact where $\phi(x)=(x, x)$ and $\theta(x, y)=x-y$. Since $\mathscr{F} J_{n}$ and $\left(\mathscr{F} J_{n-1}\right) e \Lambda$ are isomorphic to right ideals of $e \Lambda e$ and $\Lambda$ respectively, therefore Lemmas 1 and 2 complete the proof.

THEOREM 1. Let $\Lambda=\left(m^{\lambda_{i j}}\right)$ be a triangular order in $M_{n}(K)$. Then the following are equivalent: (1) gl. $\operatorname{dim} . \Lambda<\infty$, (2) $\Omega_{n} \subseteq \Lambda$, (3) gl. dim. $\Lambda \leqq$ $n-1$.

Proof. $\quad(1) \Rightarrow(2)$. Proceed by induction on $n$. For $n=2$, the result is trivial and known [5]. Assume $n>2$. If $\lambda_{i, i-1 \neq 0}$ for $2 \leqq i \leqq n$ then, by Lemma 5, $\lambda_{n, n-1}=1$. So, Proposition 1 shows that gl. $\operatorname{dim} . e \Lambda e<\infty$. Hence by induction hypothesis $\Omega_{n-1} \subseteq e \Lambda e$. Since $\lambda_{n, n-1}=1$, therefore $\Omega_{n} \subseteq \Lambda$. If $\lambda_{l, l-1}=0$ for some $l$, then $\Lambda$ is Morita equivalent to $f \Lambda f$, where $f=\sum_{i=1 ; i \neq l}^{n} e_{i i}$. By induction hypothesis $\Omega_{n-1} \subseteq f \Lambda f \subseteq M_{n-1}(K)$, so $\Omega_{n} \subseteq \Lambda$ by Lemma 6 .

$(2) \Rightarrow(3)$. Again we put an induction on $n$. For $n=2$, the result is true and trivial [5]. Let $n>2$. If $\lambda_{i, i-1} \neq 0$ for $2 \leqq i \leqq n$, then Lemma 6 and the induction hypothesis show that gl. dim. $e \Lambda e \leqq n-2$. By Proposition 1, gl. dim. $\Lambda \leqq n-1$. If $\lambda_{l, l-1}=0$ for some $l$, then $\Lambda$ is Morita equivalent to $f \Lambda f$. By Lemma $6, \Omega_{n-1} \subseteq f \Lambda f \subseteq M_{n-1}(K)$, so, by induction hypothesis, gl. $\operatorname{dim} . \Lambda=$ gl. dim. $f \Lambda f \leqq n-2$.

$(3) \Rightarrow(1)$. Clear. This completes the proof.

Proposition $1^{\prime}$. If $\lambda_{i, i-1 \neq} \geqslant 0$ for $2 \leqq i \leqq n$ and if $\lambda_{2,1}=1$ then gl. $\operatorname{dim} . \Lambda<$ $\infty$ if and only if gl. dim. $e^{\prime} \Lambda e^{\prime}<\infty$ where $e^{\prime}=\sum_{i=2}^{n} e_{i i}$. Further, if gl. dim. $\Lambda$ $=\alpha<\infty$, gl. dim. $e^{\prime} \Lambda e^{\prime}=\gamma<\infty$, then $\gamma \leqq \alpha \leqq \gamma+1$.

Proof. Similar to Proposition 1. 
We now look at the triangular orders in $M_{n}(K)$ with global dimension $n-1$.

LEMMA 7. Let $\Lambda=\left(m^{\lambda_{i j}}\right)$ be a triangular order in $M_{n}(K), n \geqq 3$. If gl. $\operatorname{dim} . \Lambda=n-1$ then $\operatorname{hd}_{\Lambda} J_{i}=i-1$ for $1 \leqq i \leqq n-1$ and hd $_{\Lambda} J_{n}=n-3$.

Proof. By induction on $n$. For $n=3$, the only triangular order of global dimension two is $\Omega_{3}$, for which the assertion is trivial. Let $n_{\neq}>3$. Since gl. $\operatorname{dim} . \Lambda=n-1$, therefore by Theorem $1, \lambda_{i, i-1}=1$ for $2 \leqq i \leqq n$. Hence, by Proposition 1 and Theorem 1 , gl. dim. $e \Lambda e=n-2$. As seen in Lemma 3 and Proposition $1, J(e \Lambda e) \cong \bigoplus_{i=1}^{n-1} \mathscr{F} J_{i}, h_{\Lambda} J_{i}=h_{e \Lambda e} \mathscr{F} J_{i}$ if $i \neq n-1$ and $\operatorname{hd}_{\Lambda}\left(\mathscr{F} J_{n-1}\right) e \Lambda=\operatorname{hd}_{e \Lambda e} \mathscr{F} J_{n-1} ;$ by induction hypothesis, $\operatorname{hd}_{\Lambda} J_{i}=\operatorname{hd}_{e \Lambda e} \mathscr{F} J_{i}$ $=i-1$ for $1 \leqq i \leqq n-2, \quad \operatorname{hd}_{\Lambda}\left(\mathscr{F} J_{n-1}\right) e \Lambda=\operatorname{hd}_{e \Lambda e} \mathscr{F} J_{n-1}=n-4$. Since $\mathscr{F} J_{n}$ is isomorphic to a right ideal of $e \Lambda e$, therefore $\mathrm{hd}_{\Lambda} J_{n}=\mathrm{hd}_{e \Lambda e} \mathscr{F} J_{n} \leqq$ $n-3$, by Lemma 2 . By hypothesis gl. dim. $\Lambda=n-1$. Hence, by Lemma $1, \operatorname{hd}_{\Lambda} J_{n-1}=n-2$. So, by (*) in Proposition $1, \mathrm{hd}_{\Lambda} J_{n}=n-3$. This completes the proof.

THEOREM 2. Let $\Lambda=\left(m^{\lambda_{i j}}\right)$ be a triangular order in $M_{n}(K)$, where $n \geqq 4$. Then gl. dim. $\Lambda=n-1$ if and only if $\lambda_{i, i-1}=1, \lambda_{i, i-2}=2=\lambda_{i, i-3}$ for $2 \leqq i \leqq n$.

ProOF. For the "only if" part, we proceed by induction on $n$. Let $n=4$. Since gl. dim. $\Lambda=3$, therefore by Theorem $1, \lambda_{i, i-1}=1$ for $2 \leqq i \leqq 4$. By Propositions 1, $1^{\prime}$ and Theorem 1, gl. dim. $e \Lambda e=$ gl. $\operatorname{dim} . e^{\prime} \Lambda e^{\prime}=2$. Hence $\lambda_{3,1}=2=\lambda_{4,2}, \lambda_{4,1}=2$ or 3 . But gl. dim. $\Omega_{4}=2$ [5]; so, we must have $\lambda_{4,1}=2$. Now let $n \neq 4$. Since gl. $\operatorname{dim} . \Lambda=n-1$, therefore by Theorem 1 and Propositions 1, 1', gl. dim. $e \Lambda e=$ gl. $\operatorname{dim} . e^{\prime} \Lambda e^{\prime}=n-2$. Now the induction hypothesis completes the proof.

For the "if" part again we put induction on $n$. The assertion is easily seen to be true for $n=4$. Now let $n \neq 4$. By induction hypothesis, we have gl. dim. $e \Lambda e=n-2$. So, Lemma 7 and its proof yield $h_{\Lambda} J_{i}=i-1$ for $1 \leqq i \leqq n-2, \operatorname{hd}_{\Lambda} J_{n} \leqq n-3$ and $\mathrm{hd}_{\Lambda}\left(\mathscr{F} J_{n-1}\right) e \Lambda=n-4$. Hence, by the exact sequence $(*)$ it is enough to prove that $\mathrm{hd}_{\Lambda} J_{n}=n-3$.

Let $M$ be the right $\Lambda$-module obtained from $P_{n}$ by replacing the last two entries by $m^{2}$. By hypothesis $\lambda_{n, n-2}=2=\lambda_{n, n-3}$. So the last four entries in $M$ are equal, viz. $m^{2}$. Clearly, as in Lemma $3(\mathrm{~b}), \mathscr{G} \mathscr{F} M \cong M$, so by Lemma 3 , hd $M=\operatorname{hd}_{c \Lambda e} \mathscr{F} M \leqq n-3$. The last inequality follows by observing that $\mathscr{F} M$ is isomorphic to a right ideal of $e \Lambda e$. Repeating this two more times, we get $\mathrm{hd}_{\Lambda} M \leqq n-5$. Clearly $M+t P_{n-1}=J_{n}$ and $M \cap t P_{n-1} \cong\left(\mathscr{F} J_{n-1}\right) e \Lambda$. By the above, hd ${ }_{\Lambda}\left(\mathscr{F} J_{n-1}\right) e \Lambda=n-4$. Hence, hd $J_{n}=n-3$. This completes the proof.

Now, we give examples of successive triangular orders in $M_{2 n+1}(K)$ whose dimensions differ exactly by $n$. This disproves a conjecture of R. B. 
Tarsey [5]. We define two families $\Lambda_{2 n+1}$ and $\Gamma_{2 n+1}, n \geqq 1$, of triangular orders in $M_{2 n+1}(K)$ such that $\Lambda_{2 n+1}$ and $\Gamma_{2 n+1}$ are successive, gl. dim. $\Lambda_{2 n+1}=$ $n$ and gl. dim. $\Gamma_{2 n+1}=2 n$.

For $n=1$,

For $n=2$,

$$
\Lambda_{3}=\left(\begin{array}{lll}
R & R & R \\
m & R & R \\
m & m & R
\end{array}\right), \quad \Gamma_{3}=\left(\begin{array}{lll}
R & R & R \\
m & R & R \\
m^{2} & m & R
\end{array}\right) .
$$

$$
\Lambda_{5}=\left(\begin{array}{lllll}
R & R & R & R & R \\
m & R & R & R & R \\
m^{2} & m & R & R & R \\
m^{2} & m & m & R & R \\
m n^{3} & m & m \iota^{2} & m & R
\end{array}\right), \quad \Gamma_{5}=\left(\begin{array}{lllll}
R & R & R & R & R \\
m & R & R & R & R \\
m^{2} & m & R & R & R \\
m^{2} & m^{2} & m & R & R \\
m^{3} & m^{2} & m^{2} & m & R
\end{array}\right) .
$$

It is easy to see that gl. dim. $\Lambda_{3}=1$, gl. dim. $\Gamma_{3}=2$, gl. dim. $\Lambda_{5}=2$, gl. dim. $\Gamma_{5}=4, \Lambda_{3}$ and $\Gamma_{3}$ are successive and $\Lambda_{5}$ and $\Gamma_{5}$ are successive.

For $n \geqq 3$, let $U_{n}$ be a triangular order in $M_{n}(K)$ in which all the entries on the main subdiagonal are $m$ and all the entries below the main subdiagonal are $m^{2}$. Let $V_{n, n+1}=\left(m^{\theta_{i i}}\right)$, where $\theta_{i j}$ are as specified below:

(a) $\theta_{1, n}=\theta_{1, n+1}=1 ; \theta_{i, n}=\theta_{i, n+1}=2$ for $2 \leqq i \leqq n$.

(b) $\theta_{1, n-1}=2 ; \theta_{i, n-1}=3$ for $2 \leqq i \leqq n$.

(c) $\theta_{1, j}=3$ for $1 \leqq j \leqq n-2$.

(d) All the remaining $\theta_{i, j}=4$.

Let

$$
\Lambda_{2 n+1}=\left(\begin{array}{ll}
U_{n+1} & M_{n+1, n}(R) \\
V_{n, n+1} & U_{n}
\end{array}\right)
$$

and $\Gamma_{2 n+1}$ is obtained from $\Lambda_{2 n+1}$ by replacing $(n+2, n)$ th entry $m$ by $m^{2}$. Trivially, $\Lambda_{2 n+1}$ and $\Gamma_{2 n+1}$ are successive.

By Theorem 2, gl. dim. $\Gamma_{2 n+1}=2 n$. We claim gl. dim. $\Lambda_{2 n+1}=n$. Let $P_{i}$ and $J_{i}$ denote the $i$ th row of $\Lambda_{2 n+1}$ and its Jacobson radical. Clearly, $J_{1} \cong P_{2}$. Hence, hd $J_{1}=0$. Since

(\#) $\quad t P_{i-1}+P_{i+1}=J_{i}, \quad t P_{\imath-1} \cap P_{i+1} \cong J_{i-1}$ for $2 \leqq i \leqq n$,

therefore, by induction it follows that hd $J_{i}=i-1$ for $2 \leqq i \leqq n$. Since $t P_{n}+P_{n+3}=J_{n+2}, t P_{n} \cap P_{n+3} \cong P_{n+2}$, therefore hd $J_{n+2}=1$. Now observing that (\#) holds for $n+3 \leqq i \leqq 2 n$, we get, by induction, hd $J_{i}=i-n-1$ for $n+3 \leqq i \leqq 2 n$.

Let $M_{i}=t P_{1}+P_{i+1}$ for $2 \leqq i \leqq n-1$. Clearly $t P_{1} \cap P_{i+1} \cong M_{i-1}$ for $3 \leqq$ $i \leqq n-1$ and $M_{2}=J_{2}$. Hence, by induction, hd $M_{i}=i-1$ for $2 \leqq i \leqq n-1$. But $t M_{n-1}+P_{n+2}=J_{n+1}$ and $t M_{n-1} \cap P_{n+2} \cong P_{n}$. Therefore, hd $J_{n+1}=n-2$. 
Let $N_{i}=t P_{n}+P_{n+i}$ for $3 \leqq i \leqq n$. It is easy to see that $t P_{n} \cap P_{n+i} \cong N_{i-1}$ for $4 \leqq i \leqq n$ and $N_{3}=J_{n+2}$. Therefore, by induction, hd $N_{i}=i-2$ for $3 \leqq i \leqq n$. Since $J_{2 n+1} \cong N_{n}$, therefore hd $J_{2 n+1}=n-2$. Hence, hd $J\left(\Lambda_{2 n+1}\right)=n-1$. Therefore, by Lemma 1, gl. dim. $\Lambda_{2 n+1}=n$. This completes the proof of our claim.

REMARK. Using the usual arguments about localization and completion, it is easy to see that our results hold when $R$ is a Dedekind domain rather than DVR.; cf. [5].

\section{BIBLIOGRAPHY}

1. Vasanti A. Jategaonkar, Global dimension of triangular orders over DVR, Notices Amer. Math. Soc. 18 (1971), 626. Abstract \#71T-A107.

2. Bruno J. Mueller, On semi-perfect rings, Illinois J. Math. 14 (1970), 464-467. MR 41 \#6909.

3. Joseph J. Rotman, Notes on homological algebra, Van Nostrand Reinhold, New York, 1968.

4. L. Silver, Noncommutative localizations and applications, J. Algebra 7 (1967), 44-76. MR 36 \#205.

5. R. B. Tarsey, Global dimension of orders, Trans. Amer. Math. Soc. 151 (1970), 335-340.

6. - , Global dimension of triangular orders, Proc. Amer. Math. Soc. 28 (1971), $423-426$.

Department of Mathematics, Cornell University, Ithaca, New York 14850 